\title{
Experimental Signatures of the Quantum-Classical Transition in a Nanomechanical Oscillator Modeled as a Damped Driven Double-Well Problem
}

\author{
Qi Li, Arie Kapulkin, Dustin Anderson, Shao Min Tan, Arjendu K. Pattanayak \\ Carleton College, 1 College Street, Northfield, Minnesota, 55057
}

\begin{abstract}
We demonstrate robust and reliable signatures for the transition from quantum to classical behavior in the position probability distribution of a damped double-well system using the Qunatum State Diffusion approach to open quantum systems. We argue that these signatures are within experimental reach, for example in a doubly-clamped nanomechanical beam.
\end{abstract}

PACS numbers: PACS numbers: 29.25.Bx. 41.75.-i, 41.75.Lx 


\section{INTRODUCTION}

The difference between quantum and classical behavior is of fundamental interest with many experimental consequences in contexts such as superconducting quantum interference devices (SQUIDs), cold atom systems, and significantly in nanoelectromechanical systems (NEMS). NEMS are small, oscillate at high frequencies and are typically maintained at low temperatures. Most of their parameters are adjustable, making them an ideal setting to explore the transition from quantum to classical behavior. Recent experimental results 1 , 2], indicate that such systems can now be prepared and observed in their ground state, thus enabling the study of genuinely non-classical behavior in the dynamics. The exciting prospect of being able to observe the quantum-classical transition in detail has contributed to interest in quantum mechanical nanoelectromechanical systems (occasionally refered to as QEMS in the literature), whose study is an active and rapidly expanding field of research [3].

A seminal theoretical study of the quantum-classical transition in NEMS was carried out by Katz et al[4] using a nonlinear resonator, which is a paradigmatic model for several common types of NEMS that yields quantum behavior distinct from the classical. They considered an isolated resonator as well as one modeled as an open system (coupled to the environment) in search of experimental signatures of such quantum behavior. Specifically, they examined the dynamics of the classical phase space distribution in such a system compared to that of the quantum-mechanical Wigner function, and looked at a zero temperature and a finite temperature environment. They were able to find differences in experimentally accessible signatures: In particular, the quantum mechanical version of the resonator has non-zero probability of being found in a position where the classical resonator has zero probability of being found. However, these differences are relatively small and blur away quickly when experimental noise or finite temperature effects through thermal noise are considered.

In this paper, by considering a slightly different but equally accessible nonlinear oscillator, we are able to obtain more pronounced experimental signatures of the quantum-classical transition; these are arguably more visible at finite temperatures and in the presence of experimental noise. Further, we show that rather than comparing just quantum and classical behavior, there is insight to be gained from studying the continuous transition where the classical behavior emerges as the limiting case of the quantum dynamics. That is, although systems are fundamentally described by quantum mechanics, their behavior changes as we 
increase the size of the system (affecting the characteristic action scaled by $\hbar$ ), the effect of the environment (decoherence) or other parameters of the system. For our system, we indeed recover the classical behavior as the limiting case. The details of the emergence of the classical limit are illuminating, and in particular, the signatures do not always change monotonically, thus establishing that comparing a single quantum solution with a classical solution leads to an incomplete picture.

Katz et al considered the sytem with the Hamiltonian

$$
H_{\text {sys }}=\frac{1}{2} p^{2}+\frac{1}{2} x^{2}+\frac{1}{4} x^{4}-x F \cos \omega t
$$

This quadratic nonlinear oscillator is within experimental reach in the near-quantum regime, and is an excellent system to study. However, even more interesting effects such as quantum tunneling obtain if we change the sign so that the $x^{2}$ term is negative[5]. This yields the so-called double-well Duffing oscillator, which has the Hamiltonian $H_{\text {sys }}=\frac{1}{2} p^{2}-\frac{1}{2} x^{2}+$ $\frac{1}{4} x^{4}-x F \cos \omega t$, and allows for clearer signatures of the classical to quantum transition as we demonstrate below.

This paper is organized as follows: We start by motivating the modeling of a specific NEMS as a doubly-clamped beam to show the connection between experimental paramters and model parameters. We then sketch the quantum state diffusion (QSD) approach, where Lindblad operators act within stochastic Schrodinger equations to incorporate the effect of the environment, used to model the behavior of such an oscillator understood as an open quantum system. These two allow us to establish that changing the sign of the quadratic term is within current experimental capabilities and that such experiments are in or close to the realm where the quantum to classical transition can be explored. We then present results and conclude with our analysis.

\section{NEMS MODELED AS A DOUBLY-CLAMPED BEAM}

Many nanoelectromechanical devices can be modeled as doubly-clamped beams driven near resonance and continuum mechanics continues to serve as an adequate model even at the submicron scale[ 6 - 8$]$. Usually magnetomotive[9] or optical[ 6 ] actuation is used to study

the resonance behaviors of NEMS such as dynamically induced bistability, hysteresis and 
effects of parameter attenuation on energy dissipation. The resonant behavior of the doublyclamped NEMS structure has been experimentally identified with the fundamental bending mode[10] of the problem. We sketch here the theory allowing us to relate the resonance frequency to characteristic parameters of NEMS and to determine the scale of the system with respect to $\hbar$, establishing the regime of the transition to classical behavior in these systems.

Starting with the assumption of an ideal beam of rectangular cross-sectional area, we can obtain the potential energy $V$ of a doubly-clamped beam under tension along the longitudinal direction. This consists of two parts[11]: elastic and bending potential energy,

$$
V_{\text {elastic }}=T\left(\int_{0}^{l_{0}} \sqrt{1+\left(y^{\prime}\right)^{2}} d x-l_{0}\right)
$$

and

$$
V_{\text {bending }}=\frac{E I}{2} \int_{0}^{l_{0}}\left(y^{\prime \prime}\right)^{2} d x
$$

$T$ is the external force on the beam in the form of the tension in the longitudinal direction along the beam, $E$ is the elastic modulus, $I$ is moment of inertia of the cross-section and $l_{0}$ is separation of two ends that clamp the beam. Note that $l_{0}$ need not be the length of the beam when it is neither stretched nor compressed because a static doubly-clamped beam may already be buckled due to inherent tension along the beam. $V_{\text {elastic }}$ is due to tension built up in the beam, which causes the elastic beam to deform and therefore gain energy. This is usually referred to as strain energy [12] while $V_{\text {bending }}$ is purely due to bending. The quantity EI, termed "flexural rigidity", denotes the force necessary to bend a beam by a unit of curvature.

The tension $T$ along the beam has two parts[13]: firstly, inherent tension $T_{0}$ (either from compression at both ends of the beam or manufacturing process) and additional tension $\Delta T$ (due to transverse motion that stretches the beam), whence $T=T_{0}+\Delta T$. When the beam is stretched, its length changes and therefore the additional tension $\Delta T$ is given by fractional change in length multiplied by elastic modulus and cross section area $A[13]: \Delta T=\frac{l-l_{0}}{l_{0}} E A$, where $l$ refers to the actual length of the buckled beam. We can rewrite this length as

$$
l=l_{0}+\Delta l=\int_{0}^{l_{0}} d x \sqrt{1+\left(y^{\prime}\right)^{2}} \approx \int_{0}^{l_{0}} d x\left(1+\frac{1}{2}\left(y^{\prime}\right)^{2}\right)=l_{0}+\frac{1}{2} \int_{0}^{l_{0}}\left(y^{\prime}\right)^{2} d x
$$


This yields that the change in tension $\Delta T=\frac{E A}{2 l_{0}} \int_{0}^{l_{0}}\left(y^{\prime}\right)^{2} d x$ whence the total tension

$$
T=T_{0}+\frac{E A}{2 l_{0}} \int_{0}^{l_{0}}\left(y^{\prime}\right)^{2} d x .
$$

Substituting these expressions for $\Delta l$ and $T$ in the expression for elastic potential energy above, we get

$$
V_{\text {elastic }}=\frac{T_{0}}{2} \int_{0}^{l_{0}}\left(y^{\prime}\right)^{2} d x+\frac{E A}{4 l_{0}}\left[\int_{0}^{l_{0}}\left(y^{\prime}\right)^{2} d x\right]^{2} .
$$

Thus, the total potential energy is

$$
\begin{aligned}
V & =V_{\text {elastic }}+V_{\text {bending }} \\
& =\frac{T_{0}}{2} \int_{0}^{l_{0}}\left(y^{\prime}\right)^{2} d x+\frac{E A}{4 l_{0}}\left[\int_{0}^{l_{0}}\left(y^{\prime}\right)^{2} d x\right]^{2}+\frac{E I}{2} \int_{0}^{l_{0}}\left(y^{\prime \prime}\right)^{2} d x
\end{aligned}
$$

At low temperatures, as is often true for NEMS experiments, it is sufficient to consider only the fundamental mode. Mode amplitude solutions for this fundamental mode $y(x)$ are of the form [14] $y(x)=\frac{1}{2} Y\left[1-\cos \left(2 \pi \frac{x}{l_{0}}\right)\right]$, where $Y$ is the transverse displacement of the center point of the beam. When we make this substitution, the fundamental mode amplitude $Y-$ that is, the location of the vibrating central point - is effectively the position variable of a single particle evolving in a potential $V$, which as a function of $Y$ is given by

$$
\begin{aligned}
V(Y) & =\frac{E I \pi^{4}}{l_{0}^{3}} Y^{2}+\frac{\pi^{2} T_{0}}{4 l_{0}} Y^{2}+\frac{E A \pi^{4}}{16 l_{0}^{3}} Y^{4} \\
& =Y^{2}\left(\frac{E I \pi^{4}}{l_{0}^{3}}+\frac{\pi^{2} T_{0}}{4 l_{0}}\right)+Y^{4} \frac{E A \pi^{4}}{16 l_{0}^{3}} .
\end{aligned}
$$

Here $I$, the moment of inertia of rectangular cross section is $\frac{a b^{3}}{12}[15]$, where $a$ is the thickness of the beam and $b$ is its width. $I$ varies with the geometry of the beam for specific experimental setups. Further, depending on whether inherent tension $T_{0}$ compresses or stretches the beam, the sign of $T_{0}$ can be negative or positive. Compressive inherent tension along the beam can arise from manufacturing processes, and can vary from $200 \mathrm{MPa}$ to $1 \mathrm{GPa}[14]$. In general, the inherent tension is not known a priori in an experimental setup. Instead, by considering the continuum mechanics model and fitting the experimentally measurable resonance frequency, the inherent tension can be calculated[10]. For sufficiently large inherent compressive tension, $T_{0}<-\frac{4 \pi^{2} E I}{I_{0}^{2}}$, we obtain a double-well potential and the beam is in a state of "Euler instability" [8, 16], meaning that it is buckled. 
Thus a single-well potential or a double-well potential can be experimentally achieved in doubly-clamped NEMS structures and these systems can then be studied for their dyamical behavior. The dynamics of a single-well NEMS actuated near the fundamental bending mode

frequency, has been extensively studied[17, 18] but that of the double-well case has not been yet been fully explored. The results of Ref. [10] suggest that it within experimental reach and the above clarifies that in general tuning the characteristic parameters should enable switching from a single-well to a double-well potential.

\section{OPEN QUANTUM SYSTEMS}

We now turn to the quantum version of the model for the problem and connect characteristic parameters with the degree of 'quantumness' of the system. An open quantum system with weak system-environment coupling and a Markovian environment is modeled by a master equation

$$
\begin{aligned}
\dot{\hat{\rho}}(t)= & \frac{-i}{\hbar}[\hat{H}, \hat{\rho}]-\frac{1}{2} \sum_{j}\left({\hat{L_{j}}}^{\dagger}{\hat{L_{j}}}_{j} \hat{\rho}+\hat{\rho}{\hat{L_{j}}}^{\dagger} \hat{L}_{j}\right) \\
& +\sum_{j}{\hat{L_{j}}}_{j} \hat{L_{j}}{ }^{\dagger}
\end{aligned}
$$

where $\hat{L}_{j}$ is the Lindblad operator representing the system-environment interaction and $\hat{\rho}$ is the reduced density operator. The quantum state diffusion (QSD) approach corresponds to solving quantum trajectories which are unravellings of the master equation. This allows for numerical efficiencies, and physical insights not available via the master equation. Specifically, we can use the QSD numerical library[19] to solve a stochastic version of the Schrödinger equation

$$
\begin{aligned}
d|\psi\rangle= & \frac{i}{\hbar} \hat{H}|\psi\rangle d t \\
& +\sum_{j}\left(\left\langle\hat{L}_{j}^{\dagger}\right\rangle{\hat{L_{j}}}-\frac{1}{2}{\hat{L_{j}}}^{\dagger}{\hat{L_{j}}}-\frac{1}{2}\left\langle{\hat{L_{j}}}^{\dagger}\right\rangle\left\langle\hat{L_{j}}\right\rangle\right)|\psi\rangle d t \\
& +\sum_{j}\left({\hat{L_{j}}}-\left\langle\hat{L}_{j}\right\rangle\right)|\psi\rangle d \xi_{j} .
\end{aligned}
$$


The solution $|\psi(t)\rangle$ to Eq. (10) is a "quantum trajectory", and we obtain $\hat{\rho}=\frac{1}{M} \sum_{i}^{M}\left|\psi^{i}\right\rangle\left\langle\psi^{i}\right|$, as the mean over an ensemble of $M$ normalized pure state projection operators[19]. This $\hat{\rho}$ satisfies the Lindblad master equation Eq. (9). That is, starting with an ensemble of identical pure states, we obtain the time evolution of multiple trajectories that evolve into an ensemble of different pure states due to interaction with the environment; this is the density matrix.

For the open quantum double-well Duffing oscillator $\hat{H}$ and $\hat{L_{j}}$ in Eq. (10) are chosen as [4, 20-22]

$$
\begin{aligned}
& \hat{H}=\hat{H}_{D}+\hat{H}_{R}+\hat{H}_{e x} \\
& \hat{H}_{D}=\frac{1}{2 m} \hat{p}^{2}+\frac{m \omega_{0}^{2}}{4 l^{2}} x^{4}-\frac{m \omega_{0}^{2}}{2} x^{2}, \\
& \hat{H}_{R}=\frac{\gamma}{2}(\hat{x} \hat{p}+\hat{p} \hat{x}) \\
& \hat{H}_{e x}=-g m l \omega_{0}^{2} \hat{x} \cos (\omega t) \\
& {\hat{L_{1}}}=\sqrt{\gamma(1+\bar{n})}\left(\sqrt{\frac{m \omega_{0}}{\hbar}} \hat{x}+i \sqrt{\frac{\gamma}{m \omega_{0} \hbar}} \hat{p}\right), \\
& \hat{L}_{2}=\sqrt{\gamma \bar{n}}\left(\sqrt{\frac{m \omega_{0}}{\hbar}} \hat{x}-i \sqrt{\frac{\gamma}{m \omega_{0} \hbar}} \hat{p}\right) .
\end{aligned}
$$

where $\gamma$ is the strength of coupling between the oscillator and thermal bath environment. We now define $\hat{x}$ and $\hat{p}$ in unitless forms $\hat{Q}=\sqrt{m \omega_{0} / \hbar} \hat{x}$ and $\hat{P}=\sqrt{1 / m \omega_{0} \hbar} \hat{p}$ respectively [21, 22] yielding the dimensionless set of equations

$$
\begin{aligned}
& \hat{H_{D}^{\prime}}=\frac{1}{2} \hat{P}^{2}+\frac{\beta^{2}}{4} \hat{Q}^{4}-\frac{1}{2} \hat{Q}^{2} \\
& \hat{H}_{R}^{\prime}=\frac{\Gamma}{2}(\hat{Q} \hat{P}+\hat{P} \hat{Q}) \\
& \hat{H}_{e x}^{\prime}=-\frac{g}{\beta} \hat{Q} \cos (\Omega t) \\
& \hat{L_{1}^{\prime}}=\sqrt{\Gamma(1+\bar{n})}(\hat{Q}+i \hat{P}) \\
& \hat{L_{2}^{\prime}}=\sqrt{\Gamma \bar{n}}(\hat{Q}-i \hat{P})
\end{aligned}
$$

where the time in measured in units of $\omega_{0}^{-1}$ so that $\Gamma=\gamma / \omega_{0}, \Omega=\omega / \omega_{0}$. In Eqs. (15, 16]20,21), $\bar{n}=\left(e^{\hbar \Omega / k_{B} T}-1\right)^{-1}$ is the Bose-Einstein distribution of thermal photons representing the effect of the environment at finite $T$ and is evaluated at the natural frequency of the oscillator[23]. This dimensionless formulation isolates the scaling factor 
$\beta=\sqrt{\frac{\hbar}{S}}$ as the single length scale of the problem, where $S$ is the classical action, such that $\beta^{2}=\frac{\hbar}{m l^{2} \omega_{0}}$. For the classical equation of motion for a damped Duffing oscillator, which is $\frac{d^{2} x}{d t^{2}}+2 \Gamma \frac{d x}{d t}+\beta^{2} x^{3}-x=\frac{g}{\beta} \cos (\Omega t)$, solutions to the classical equation of motion are unchanged with respect to different values of $\beta$ except for the length scale of the phase space. However, changing $\beta$ changes the quantum solution considerably.

Comparing the quartic potential Eq. (8) for the motion of the fundamental mode of the nanomechanical resonator with the potential term in the Duffing Hamiltonian Eq. (12), it is straightforward to see that for the quantum nanoelectromechanical resonator

$$
\beta^{2} \equiv \frac{\hbar}{S}=\frac{\hbar l_{0}}{8 \pi^{2}} \sqrt{\frac{A}{2 E \rho I^{3}\left[(-1)\left(1+\frac{T_{0} l_{0}^{2}}{4 \pi^{2} E I}\right)\right]^{3}}}
$$

where, as noted in section II, we restrict our consideration to resonators of the double well shape with $T_{0}<-\frac{4 \pi^{2} E I}{l_{0}^{2}}$. The scaling parameter $\beta^{2}$ determines the way in which the action of the nanomechanical system scales relative to $\hbar$.

As such, studying the system as $\beta$ is changed allows us to explore the quantum to classical transition. Previous theoretical work [20 22] for the Duffing problem focused on the behavior of the quantum trajectories has confirmed that $\beta \rightarrow 0$ indeed recovers the classical limiting behavior, including the presence of 'strange attractors' of chaos in the phase space, while $\beta=1$ shows behavior distinctive of the quantum regime. There is interesting physics in the intermediate regime[20], and in particular the details of the transition as $\beta$ changes are informative (as below). As shown in Table (II), doubly-clamped nanoelectromechanical beams can be made of different materials including Si [5, 14], single-walled carbon nanotubes (SWNT) or multi-walled (MWNT) nanotubes[6] and even metals like gold[24]. The analysis above for the Duffing oscillator also works for a doubly-clamped Pt nanowire[25], which has a high fundamental frequency (greater than $1 \mathrm{GHz}$ ). The characteristic parameters given in Table (II) for example show that the current experimental setups can be tuned within the range of $\beta$ considered in this paper. Specifically, for the SWNT experiment of Witkamp et al[10], (see Table I), they used a device of radius $r=1.6 \mathrm{~nm}$ and length $l_{0}=1.15 \mu \mathrm{m}$. This yields, using their other device parameters and an $I=\frac{\pi r^{4}}{4}$ that

$$
\beta^{2}=5.34 X 10^{-6} X\left(\frac{\lambda}{4 \pi^{2}}-1\right)^{-3 / 2}
$$


where $\lambda=\frac{T_{0} l_{0}^{2}}{E I}$ is greater than $4 \pi^{2}$ to yield the double-well shape. If $\lambda$ is in the range $(39.5-60)$, we get $\beta$ in the range $(0.65-0.04)$, which as we see below, is precisely the range needed to map the transition. Witkamp et al report $\lambda=26$, so this is clearly well within reach of current experiments.

TABLE I: Four common types of doubly-clamped structure of NEMS that have been realized experimentally. The characteristic parameters can be used to find the fundamental mode frequency.

\begin{tabular}{|l|l|l|l|l|}
\hline Type & Length, $l_{0}(\mathrm{~nm})$ & Diameter $(\mathrm{nm})$ & Density $\rho\left(\mathrm{kg} / \mathrm{m}^{3}\right)$ & Elastic Modulus $E(\mathrm{TPa})$ \\
\hline Si $[\underline{5}]$ & $50-10^{4}$ & $5 \times 10$ to $10 \times 20$ & 2330 & 0.137 \\
\hline SWNT[10],[6] & $50-10^{4}$ & 1 & 1930 & 1.25 \\
\hline MWNT[6] & $50-10^{4}$ & 20 & 1930 & 1.25 \\
\hline Pt nanowire[25] & 1300 & 43 & 168 & 21090 \\
\hline
\end{tabular}

There are other parameters of interest related to the system-environment interactions. In particular, the experimentally measurable quality factor $Q$ quantifies energy lost in comparison to total energy of the resonator during one complete driving period which can be related to the damping $\Gamma$. A comprehensive account for the mechanisms of quality factor, $Q$, in NEMS has not been established[24]. but for the case of doubly-clamped beam, $Q$ can arise from clamping, thermal elastic damping, as well as defects of crystalline structure of the material[7]. Usually, $Q$ can be measured experimentally using magnetomotive techniques [9]. For low temperatures (20 K and below) $Q$ can be as large as of the order of $10^{3}[17$. Lowering the temperature further to the milikelvin range further depresses energy dissipation and recent experiments[24, 26] have achieved quality factors on the order of $10^{5}$. Although these experiments have pushed towards high $Q$ and correspondingly low $\Gamma$, it is clearly possible to make systems more dissipative, thus allowing the exploration of a large range in $\Gamma$.

\section{RESULTS AND DISCUSSIONS}

The solutions to Eq. (10) yield "quantum trajectories" which have been previously discussed in detail in the context of the recovery of classical behavior from quantum mechanics[20 22]. What we focus our attention on below is ensemble-and-time-averaged 
asymptotic behavior of the system, defined through the probability distributions

$$
P_{a v g}(x)=\sum_{i=1}^{M} \sum_{k=1}^{N} \frac{1}{M N}\left\langle\psi_{k}^{i}(t) \mid \psi_{k}^{i}(t)\right\rangle
$$

with $t_{\text {transient }}+4 \pi>t>t_{\text {transient }}$. In doing this we are averaging over multiple $M$ trajectories generated in QSD simulations to yield the behavior of density matrices accessible the laboratory. We also eliminate phase-dependent idiosyncracies of these distributions and correlate better with experimental capabilities regarding the measurement of the dynamics of the quadratures by time-averaging trajectories $\left|\psi_{k}^{i}(t)\right\rangle$ with $N$ samples of each trajectory over two driving periods, taken after a suitably long transient time $t_{\text {transient }}$.

The time averaging is not necessary but is useful because (a) in exploring the quantum to classical transition, the properties that signal change most clearly as parameters are varied are asymptotic, global characteristics of the system rather than unrepresentative "snap shots" at specific times. Also (b), the time-averaging eliminates experimental difficulties in determining the phase of the driving relative to the observation, as well as allowing one to quickly build statistics.

We have explored $P_{\text {avg }}(x)$ at both zero temperature (that is, $\bar{n}=0$ ) and finite temperature for several $\beta$; we report here $\beta=0.01,0.3$ and 1.0. For each of these values, as we change the coupling strength $\Gamma$, we also looked for signatures of the quantum to classical transition through the changes in $P_{\text {avg }}(x)$. Fig. (1) shows $P_{\text {avg }}(x)$ at zero temperature. With high dissipation $(\Gamma=0.3)$ we see what we term nonmonotonicity in the behavior of the probability distribution as a function of $\beta$. Specifically, the probability distribution changes as follows: (a) At $\beta=0.01$ it is an asymmetric object centered in a single well, followed by (b) at $\beta=0.3$ an essentially symmetric double-peak structure across both wells and then (c) at $\beta=0.1$ a symmetric single peak straddling the central potential barrier.

We understand these results as follows: For $\beta=0.01$, the most classical case, the dissipation results in the particle being unable to overcome the central potential barrier even with driving and therefore being confined in a single well. The probability peaks at both ends of the probability distribution correspond to the classical turning points of the oscillator orbit in phase space. That is, the dynamics in this situation are identical to those that would be generated by a classical trajectory, and unsurprisingly this means that the probability distribution is also entirely explained by classical considerations. 
As we increase the quantum-ness of the system (decrease the scale of the system) to $\beta=0.3$, the particle acquires a nonzero probability of being in both wells. This is classically impossible given that it is a zero-temperature problem with high damping. The inter-well transitions could indeed occur because, as the system gets more quantum-mechanical with $\beta$ increasing, the scale of the Lindblad term effectively also increases. It is therefore possible that the particle continues to act essentially classically but the increased effect of the noise leads to a classical noise-activated hopping over the barrier. However, our studies show that the particle is behaving entirely quantum-mechanically during the interwell transition, whence this transition is a quantum tunneling effect. Specifically, we have seen that the Wigner function for the particle shows classically forbidden negative regions throughout its dynamics. We have also seen that the energy expectation value remains negative while the expectation value of the position transits past the origin[20]. Finally, we have found instances in time when a wave-packet has significant probability on either side of the barrier, which is incomensurate with it being a localized wavepacket behaving like a classical particle hopping over the barrier. Incidentally, the last two demonstrate one advantage of using the QSD wavefunction-based approach, since such information is unavailable in the master equation approach. The symmetric double-peak structure of $P_{\text {avg }}(x)$ at this value of $\beta$ indicates that the ensemble average over the wave packet behavior leads to an asymptotically equal probability in either well.

For $\beta=1$, the most quantum case, the probability is peaked at the origin. There are multiple ways of understanding this. The first is that the zero point energy is such that the lowest states of the potential are above the barrier. As such the probability is peaked in the middle of the quartic larger well, ignoring the quadratic bump at the bottom. Another way of understanding this situation is to realize that as $\beta$ is increased above 0.3 , the scale of the system changes such that the two peaks of the symmetric distribution from $\beta=0.3$ come closer together until they merge.

These three figures show not only that there is a clearer difference between the quantum and the classical probability behavior in the double-well Duffing oscillator compared to the single-well version, there are interesting things to be learned from studying the intermediate regime.

As as alternative case, we also present results from slightly lower dissipation, with $\Gamma=$ 0.125. In this case, even at the classical limit, the dissipation is not strong enough to confine 
the particle in one well. Specifically, the particle trajectory is that of a strange attractor across both wells[20 22] which yields the unusual shape for the probability distribution. The difference in the classical behavior does not persist, and the distributions at higher $\beta$ for this $\Gamma$ value follow the same pattern as those for $\Gamma=0.3$. As such, comparing the two sets of figures, the results at $\Gamma=0.3$ show a more dramatic transition from the classical to the quantum regime.

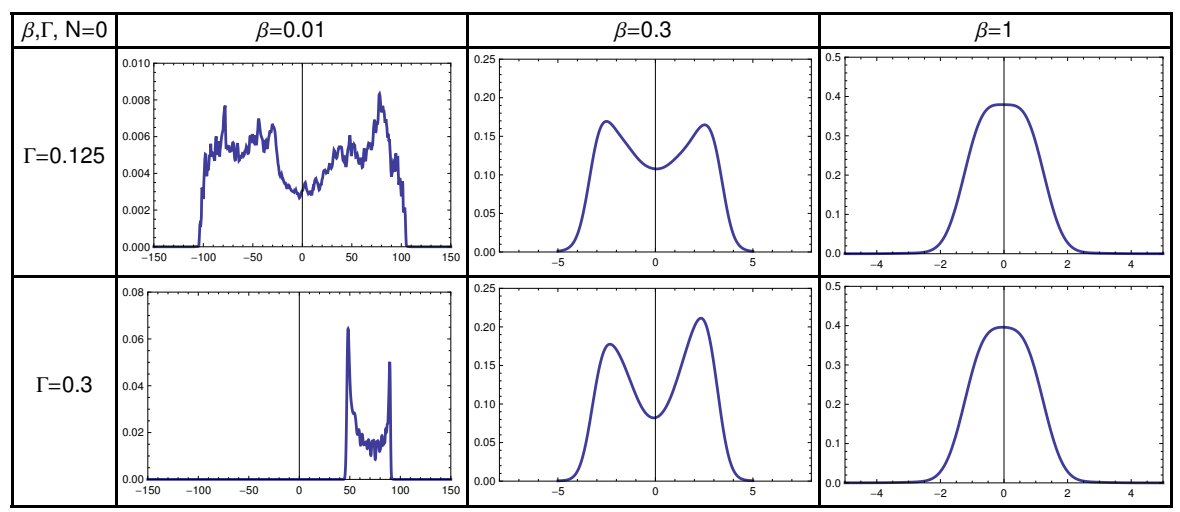

FIG. 1: $P_{\text {avg }}(x)$ plots for various parameters. The degree of quantumness $\beta$ increases from left to right as $0.01,0.3,1.0$. The values for $\Gamma=0.125,0.3$.

At even lower values of $\Gamma$, the vanishing dissipation means that the wavefunctions do not localize in a basis as happens for greater dissipation[19]. This leads to the standard semiclassical convergence problem for the calculations of $P_{\text {avg }}(x)$. However we are able to compute $P_{\text {avg }}(x)$ at finite temperature for each set of $\beta$ and $\Gamma$ and these are presented in Fig. (2). We see quickly that the results are similar to those at zero temperature in Fig. (11) though we can see that temperatures corresponding to $\simeq 4$ thermal photons begins to eliminate the structure of the distribution(s) at $\beta=0.3$; this is roughly where quantum effects are equal to thermal effects. As we can see, for large dissipation $(\Gamma=0.3)$ and the most classical case, the particle is still confined in one well; that is, thermal activation is not high enough to induce switching between wells. The probability distributions are of course broadened due to thermal effects, but the signature of the transition as $\beta$ is increased are robust. The last row of Fig. (2) reassuringly shows the existence of a smooth transition away from zero temperature behavior.

In conclusion, we have shown that in a open double-well quantum oscillator clear signatures of quantum behavior and in particular the transition away from classical behavior can be found in the changing shapes of an asymptotically obtained probability distribution 


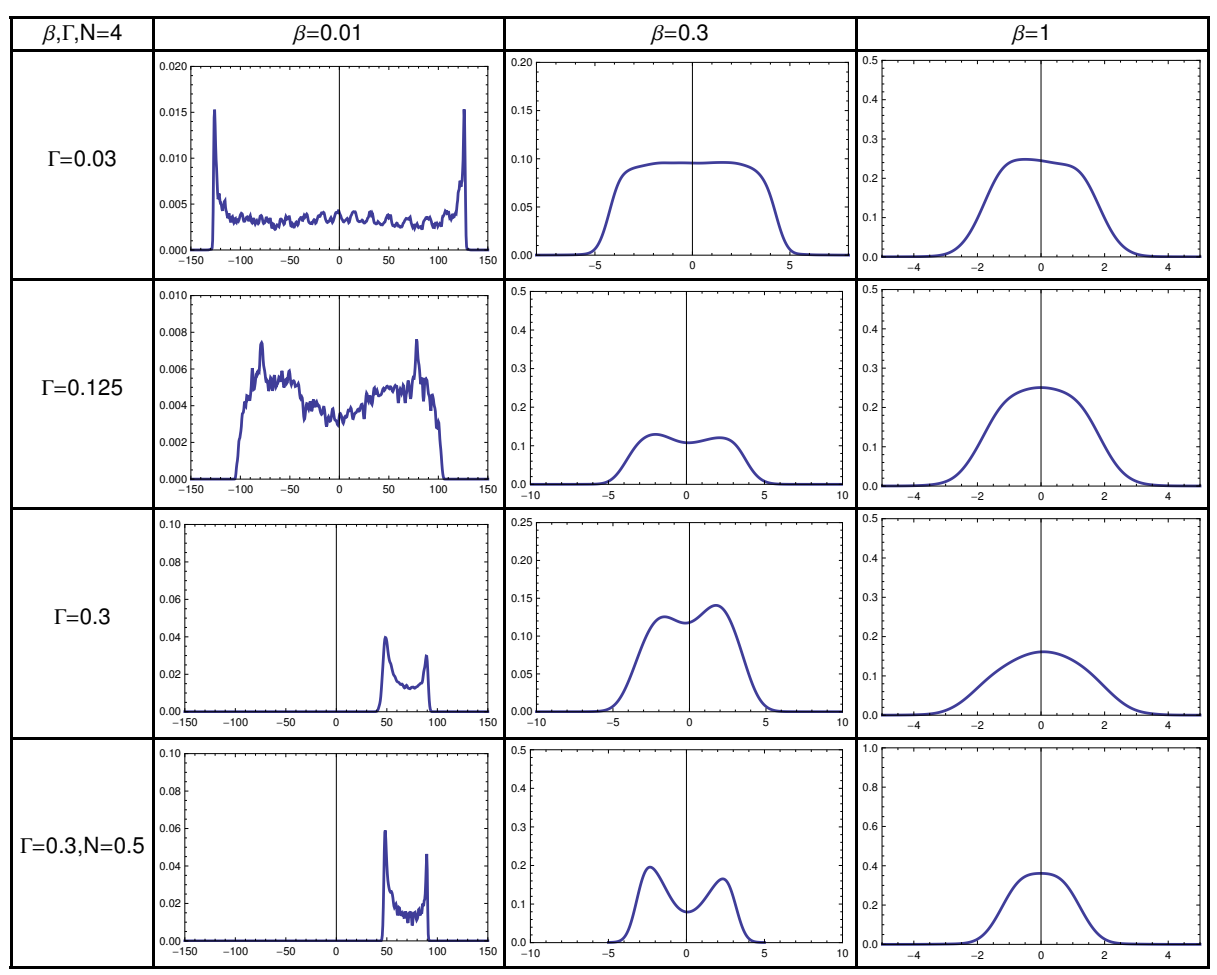

FIG. 2: $P_{\text {avg }}(x)$ plots for various parameters at finite temperature. For the first three rows, the number of thermal photons $\bar{n}=4$ and $\beta$ increases from left to right as $0.01,0.3,1.0$. $\Gamma$ increases down the grid as $0.03,0.125,0.3$. The last row has $\Gamma=0.3$ and $\bar{n}=0.5$ and shows a smooth transition from the zero temperature case in Fig. (1).

$P_{a v g}(x)$. At the right parameters, this transition from classical through quantum mechanical behavior can be nonmonotonic and hence should be clearly visible. Further, these transitions are robust at finite temperatures. The experimentally tunable parameters in NEMS allow for flexibility in exploring the parameter landscape of the transition and are excellent candidates to study this transition. We believe that such experiments are viable in doubly-clamped NEMS,(see Table.【)

Acknowledgements: The authors gratefully acknowledge funding from the Howard Hughes Medical Institute through Carleton College, and help with computing from Ryan Babbush.

[1] J. Chan, T. P. M. Alegre, A. H. Safavi-Naeini, J. T. Hill, A. Krause, S. Groblacher, M. Aspelmeyer, and O. Painter, Nature 478, 89 (Oct 2011)

[2] J. D. Teufel, T. Donner, D. Li, J. W. Harlow, M. S. Allman, K. Cicak, A. J. Sirois, J. D. Whittaker, K. W. Lehnert, and R. W. Simmonds, Nature 475, 359 (Jul 2011) 
[3] M. Poot and H. S. van der Zant, Physics Reports 511, 273 (2012)

[4] I. Katz, R. Lifshitz, A. Retzker, and R. Straub, New Journal of Physics 10, 125023 (2008)I. Katz, A. Retzker, R. Straub, and R. Lifshitz, Phys. Rev. Lett. 99, 040404 (Jul 2007)

[5] S. M. Carr, W. E. Lawrence, and M. N. Wybourne, Physical Review B 64, 22101 (2001)

[6] H. W. C. Postma, I. Kozinsky, A. Husain, and M. L. Roukes, Applied Physics Letters 86, $223105(2005)$

[7] K. L. Ekinci and M. L. Roukes, Review of Scientific Instruments 76, 061101 (2005)

[8] P. Werner and W. Zwerger, Europhysics Letters 65, 158 (2004)

[9] A. N. Cleland and M. L. Roukes, Sensors and Actuators A: Physical 72, 256 (1999)

[10] B. Witkamp, M. Poot, and H. S. J. van der Zant, Nano Letters 6, 2904 (2006)

[11] J. A. Pelesko and D. H. Bernstein, Modeling MEMS and NEMS (CRC Press, 2003)

[12] P. C. Chou and N. J. Pagano, Elasticity: tensor, dyadic, and engineering approaches (Dover Publications, 1992)

[13] R. Lifshitz and M. C. Cross, Nonlinear Dynamics of Nanosystems (Wiley-VCH, Weinheim, 2010) Chap. 8

[14] S. M. Carr and M. N. Wybourne, Appl. Phys. Lett 82, 709 (2003)

[15] L. D. Landau and E. M. Lifshitz, Theory of Elasticity (Pergamon Press, 1986)

[16] G. Weick, F. Pistolesi, E. Mariani, and F. von Oppen, Physical Review B 81, 121409 (2010)

[17] H. W. C. Postma, I. Kozinsky, A. Husain, and M. L. Roukes, Phys. Rev. Lett. 99, 207201 (2007)

[18] V. Peano and M. Thorwart, Physical Review B 70, 235401 (2004)

[19] I. Percival, Quantum State Diffusion (Cambridge University Press, 1999)

[20] A. Kapulkin and A. K. Pattanayak, Physics Review Letters 101, 074101 (2008)

[21] T. A. Brun, C. P. I, and R. Schack, J. Phys. A 29, 2077 (1996)

[22] Y. Ota and I. Ohba, Physical Review E 71, 015201 (2005)

[23] C. W. Gardiner and P. Zoller, Quantum Noise, 3rd ed. (Springer, 2004)

[24] A. Venkatesan, K. J. Lulla, M. J. Patton, A. D. Armour, C. J. Mellor, and J. R. Owers-Bradley, Physical Review B 81, 073410 (2010)

[25] A. Husain, J. Hone, H. W. C. Postma, X. M. H. Huang, T. Drake, M. Barbic, A. Scherer, and M. L. Roukes, Applied Physics Letters 83, 1240 (2003)

[26] A. K. Huttel, G. A. Steele, B. Witkamp, M. Poot, L. P. Kouwenhoven, and H. S. J. van der 
Zant, Nano Letters 9, 2547 (2009) 